\title{
Blasting Plant on Rails
}

\author{
To simplify the process of loading a blasting plant with large components, \\ the body of the plant has been designed to be moveable.
}

A major German steel construction company is responsible for planning, designing, manufacturing and assembling components and systems for bridges, industrial plants, steel buildings and hydraulic engineering facilities. The components are up to 38 metres long, which means that they are very heavy and difficult to handle.

In order to make it as easy as possible to transport these large items into a blasting plant, the manufacturer of the plant, Kiess, worked together with the steel construction company to develop a new loading system which moves the parts using trucks on a rail system and a crane. The operator chose a version of the plant designed in two halves which can be separated in the middle.

\section{Two moveable sections with a fixed side wall}

The plant consists of two moveable sections which are a total of 38 metres long, 12 metres wide and 7 metres high, with a fixed side wall. A scraper has been installed under the floor for the entire length of the blasting plant and is covered with a metal grid. Blasting takes place only over the metal grid and the blasting media is automatically transported to the recycling system by the scraper.

A bucket conveyor is used to move the blasting media up into the cleaning system. Flakes of rust, mill scale and other contamination, together with any dust, are removed by a sieving and air separation process consisting of several stages. The blasting media that is still usable is transferred to a silo, which is used for storage purposes and for filling two 200-litre blasting containers. By controlling the blasting containers it is possible to switch the supply of blasting media to the blasting unit on and off. As a result, any remaining blasting media can be removed from the edges and corners of the components and the blasting hos- es can be emptied before work is finished for the day using a fast-moving flow of air from high-performance venturi nozzles.

\section{Travelling at ten metres per minute}

Inside the blasting plant is a $10-\mathrm{mm}$ thick, wear-resistant rubber curtain which protects the plant from the blasting media and also acts as noise insulation. Highly effective LED headlights on the blasting chamber, which are protected against wear by additional lenses, and two filter units each with a throughput of $50,000 \mathrm{~m}^{3} / \mathrm{h}$ ensure that the blasting process is clearly visible and keep dust to a minimum.

The suction hoses of the filter units pass through the fixed side wall and are therefore not affected by the movement of the two parts of the blasting plant. The supply of fresh air to the plant comes from inside the building via labyrinth boxes fixed to the moving parts.

Both parts of the plant have an outer gate system consisting of 10 sections that is 12 metres wide and 7 metres high when it is opened. After the plant has moved around the workpiece, which is put in place by a gantry crane, the gate closes. The two halves of the plant are mounted on a total of eight wheel blocks, four of which are driven. The maximum speed of the blasting plant is 10 metres per minute.

\section{Precise positioning and protective buffer}

For safety reasons, there are switches on the front of each part of the plant, which ensures that the drive system can only be operated in the correct direction. This guarantees that the operator only moves each half of the plant in the direction that he can see. In addition, each part has a buffer with limit switches which stop the plant from moving if it encounters an obstacle.

The two halves of the plant must be positioned with a high level of precision in their final locations in order to move the individual elements of the gate into and out of the parking position outside the body of the plant. This is achieved by checking the position of the plant several times and automatically reducing its travelling speed. //

\section{Contact}

\section{Kiess GmbH \& Co. KG}

Mülheim/Ruhr, Germany

Tel. +4920849580

info@kiess.de

www.kiess.de
THE BRAND NEW IST WEBSITE

$\checkmark$ Top results through an optimized search engine

$\checkmark$ Direct access to all IST articles since 2010

$\checkmark$ Latest news, products and event schedule

$\checkmark$ Newsletter

www.ist-surfacetechnology.com

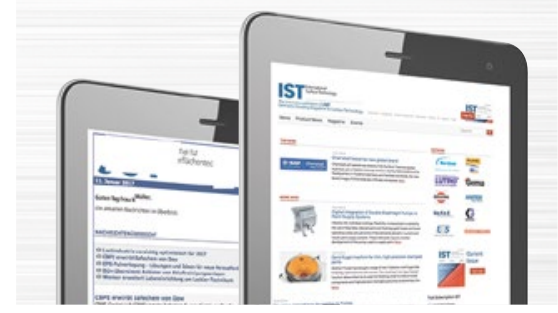

